\title{
Tarifs et conventions
}

Ernst Gähler ${ }^{a}$, Irène Marty ${ }^{b}$, Anton Prantlc

a Vice-président de la FMH, responsable du domaine Tarifs et conventions

b Cheffe Tarifs et conventions de la FMH

c Président de la direction de la Caisse des médecins

\section{Dispensation de médicaments par le médecin} La lutte pour la dispensation de médicaments par le médecin se joue sur deux plans. Premièrement, on s'efforce de rogner encore sur la marge de distribution qui passera de 15 à $12 \%$ à partir du $1^{\text {er }}$ mars 2010 suite à la décision prise par le DFI. La Commission de la sécurité sociale et de la santé publique du Conseil des Etats (CSSS-E) a d'ailleurs simultanément approuvé, en vertu du droit d'urgence, une proposition de diminution de cette marge à $8 \%$. Deuxièmement, on tente, à travers la révision de la Loi sur les produits thérapeutiques (LPTh) récemment soumise en consultation par le Conseil fédéral, de supprimer totalement la propharmacie. En effet, le projet mis en consultation a pour objectif de radier totalement ce canal de distribution et de n'admettre aux médecins uniquement la remise de médicaments couramment utilisés dans la pratique. La Confédération et les cantons peuvent prononcer des exceptions. Si l'officine publique la plus proche ne peut être atteinte par les transports publics en un temps approprié, la dispensation directe de médicaments par le médecin doit continuer à être autorisée.

Le rapport en consultation vise à remettre en question l'activité médicale et la compétence des médecins en matière d'utilisation de médicaments. Il insinue en

\section{Le corps médical s'engagera avec force pour le maintien de la propharmacie}

particulier que l'intérêt personnel du médecin risquerait de mettre en danger la sécurité des patients et des traitements. Ce rapport se fonde sur une étude de Konstantin Beck, Ute Kunze et Willy Oggier (Selbstdispensation: Kosten treibender oder Kosten dämpfender Faktor? Managed Care 6/2004), mais omet de préciser que ladite étude ne présente pas correctement les rapports de coûts réels. Par exemple, les chiffres et les calculs relatifs à la taxe de rémunération des médicaments basée sur les prestations (RBP) pour les pharmaciens n'y figurent pas. Contrairement aux médecins, les pharmaciens devraient pouvoir continuer à dispenser des médicaments soumis à ordonnance et faisant l'objet d'une désignation spécifique sans avis ou ordonnance du médecin. Cela est pourtant contraire au principe prôné par les pharmaciens que «quatre yeux valent mieux que deux».
Le groupe de travail "Dispensation directe de médicaments DMA/SD» (qui comprend les organismes suivants: FMH/VEDAG/CMPR/SSMG/CCM/FMCH/ SFSM/APA) se consacre à la question des marges et cherche des solutions pour une remise de médicaments par le médecin indépendamment de la marge.

Le groupe de travail SD/Propharmacie (qui comprend les organismes suivants: FMH/VEDAG/CCM/Médecins de famille $\mathrm{CH} / \mathrm{APA}$ ) élabore simultanément des stratégies pour le maintien de la dispensation de médicaments par le médecin et pour chaque point soumis à consultation en vue de la révision de la Loi sur les produits thérapeutiques.

L'Assemblée de délégués $(A D)$ de la FMH s'est clairement prononcée le 18 novembre 2009 en faveur du maintien de la dispensation de médicaments par le médecin indépendamment du type de rémunération. Le corps médical s'engagera avec force pour le maintien de la propharmacie.

\section{Révision de la Liste des analyses (LA)}

\section{Monitorage INFRAS/OFSP}

L'entreprise INFRAS qui a réalisé le concept de monitorage de la Liste des analyses s'occupera également de sa mise en œuvre. Pour mener à bien le monitorage «Laboratoire d'analyses au cabinet médical», l'INFRAS a besoin des données de la FMH, un accord a été signé pour régler toutes les modalités requises (gestion des données, indemnisation de la charge administrative supplémentaire, etc.). Les données de la FMH sont constituées d'informations fournies par la Caisse des médecins, NewIndex, les centres de contrôle de la qualité et les laboratoires privés (autorisés à le faire par les médecins détenteurs d'un cabinet) et par les laboratoires hospitaliers. Les données fournies permettent une analyse différenciée qui sera effectuée par Roman Fried, spécialiste en médecine de laboratoire. Le groupe d'expert qui accompagne le monitorage mis en œuvre par l'INFRAS sur mandat de l'OFSP est composé d'Anton Prantl, Roman Fried et Ernst Gähler.

\section{Monitorage élargi de la FMH}

La FMH a besoin d'un monitorage élargi pour deux raisons: premièrement, il s'agit d'évaluer l'impact objectif de la révision en matière d'économies d'entreprise, ce que l'OFSP renonce sciemment à inclure dans son monitorage. Et deuxièmement, il permettra de montrer son impact global tant sur l'ensemble du tarif TARMED que sur la Convention sur les prestations et 
les prix (LeiKoV). De plus, nous aurons également besoin d'informations différenciées adaptées spécifiquement au corps médical. Ce n'est qu'ainsi qu'il sera ensuite possible de remonter l'ensemble de la chaîne des analyses effectuées, du patient au cabinet médical (laboratoire du praticien) en passant par les laboratoires privés et hospitaliers, et d'en montrer l'impact. A ce propos, nous saisissons l'occasion pour inviter les médecins qui ne l'ont pas encore fait à remplir le coupon nous autorisant à demander leurs données anonymisées auprès de leur laboratoire d'analyses privé.

\section{Impacts - premiers résultats}

Nous continuerons à effectuer nos mesures après l'introduction de la nouvelle Liste des analyses. Les premières évaluations montrent que les médecins de premier recours et les spécialistes en médecine interne

\section{Les médecins concernés se comportent de manière exemplaire, car ils se concentrent bien plus sur la qualité des soins fournis aux patients que sur les intérêts pécuniaires}

accuseront une perte de $25 \%$ du chiffre d'affaires de leur laboratoire. Seules certaines spécialités permettront, simplement par la structure tarifaire, de générer une hausse du chiffre d'affaires pouvant atteindre $30 \%$.

Si l'on se réfère à la théorie économique, de tels écarts de chiffre d'affaires entraînent des contre-réactions pouvant à nouveau être interprétées négativement. C'est pourquoi nous avons pris en compte cet aspect dans notre monitorage portant sur plus de 4000 cabinets médicaux avec laboratoire d'analyses et plus précisément sur les médecins de premier recours et les spécialistes en médecine interne fortement concernés:

- Par le passé, la part de séances de laboratoire sans consultation était de $2,7 \%$. Sous le régime de la nouvelle Liste des analyses, ce taux est de 2,6\% actuellement. Malgré notre incitation (taxe de présence et suppléments ne pouvant être appliqués qu'en cas de consultation simultanée).

- Le rapport séance de laboratoire, consultation médicale a subi un décalage plus important. Alors que son taux était de $23,1 \%$, il ne représente plus que $21,7 \%$ aujourd'hui. Pour l'instant, on ne sait pas encore si cette baisse est due à la diminution du nombre de séances de laboratoire ou à l'augmentation des consultations médicales.

Les réactions mesurées sont donc nettement inférieures à $5 \%$. Statistiquement parlant, les médecins concernés se comportent de manière exemplaire, car ils se concentrent bien plus sur la qualité des soins fournis aux patients que sur les intérêts pécuniaires insinués par l'OFSP et la Surveillance des prix.

\section{Révision du concept de valeur intrinsèque}

Suite à d'âpres négociations, la FMH et les répondants des frais se sont mis d'accord sur une mesure aussi simple qu'efficace: le diplôme de formation continue. Désormais, la formation continue pour les positions relevant des droits acquis pourra être accomplie dans le cadre de la formation continue réglementaire et attestée au moyen du diplôme habituel de formation continue. Cette solution a été possible grâce à la révision l'année dernière de la Réglementation pour la formation continue (RFC) de la FMH. L'article de Christoph Hänggeli «Possédez-vous un diplôme de formation continue» qui paraît dans le présent numéro du BMS vous fournira plus d'informations à ce sujet.

\section{TARMED Suisse}

La révision de la structure tarifaire TARMED dépend encore et toujours de la convention sur la gestion des coûts avec les répondants des frais. Nous avons pu convenir avec santésuisse d'une procédure facilitée de révision des structures et nous nous sommes entendus sur la marche à suivre. La signature d'une convention commune permettrait toutefois d'adapter enfin la valeur du point tarifaire pour le domaine de l'assurance-accident. La conférence au sommet du 4 septembre 2009 a permis de trouver un compromis: l'augmentation de 4 centimes de la valeur du point aura lieu en deux étapes: augmentation de 1 centime au moment de la signature de la convention sur la gestion des coûts et de 3 centimes dans le cadre de la révision de la structure tarifaire.

\section{Nouvelle collaboratrice au sein du domaine «Tarifs et conventions»}

L'équipe de notre domaine est à nouveau au complet. Le 16 novembre 2009, nous y avons accueilli Mme le Dr Susanne Christen en qualité de nouvelle collaboratrice. Nous lui souhaitons une chaleureuse bienvenue. 